\title{
Endoscopic Management of Anastomotic Leakage after Esophageal Surgery: Ten Year Analysis in a Tertiary University Center
}

\author{
Nader El-Sourani', Sorin Miftode', Maximilian Bockhorn ${ }^{1}$, Alexander Arlt ${ }^{2}$, and Christian Meinhardt ${ }^{2}$ \\ ${ }^{1}$ Department for General and Visceral Surgery, University Hospital, Klinikum Oldenburg AöR, ${ }^{2}$ Department for Internal Medicine and \\ Gastroenterology, University Hospital, Klinikum Oldenburg AöR, Germany
}

Background/Aims: Anastomotic leakage after esophageal surgery remains a feared complication. During the last decade, management of this complication changed from surgical revision to a more conservative and endoscopic approach. However, the treatment remains controversial as the indications for conservative, endoscopic, and surgical approaches remain non-standardized. Methods: Between 2010 and 2020, all patients who underwent Ivor Lewis esophagectomy for underlying malignancy were included in this study. The data of 28 patients diagnosed with anastomotic leak were further analyzed.

Results: Among 141 patients who underwent resection, 28 (19.9\%) developed an anastomotic leak, eight (28.6\%) of whom died. Thirteen patients were treated with endoluminal vacuum therapy (EVT), seven patients with self-expanding metal stents (SEMS) four patients with primary surgery, one patient with a hemoclip, and three patients were treated conservatively. EVT achieved closure in $92.3 \%$ of the patients with a large defect and no EVT-related complications. SEMS therapy was successful in clinically stable patients with small defect sizes.

Conclusions: EVT can be successfully applied in the treatment of anastomotic leakage in critically ill patients, while SEMS should be limited to clinically stable patients with a small defect size. Surgery is only warranted in patients with sepsis with graft necrosis. Clin Endosc 2022;55:58-66

Key Words: Anastomotic leak; Endoluminal vacuum therapy; Esophagus; Intrathoracic; Self-expanding metal stents

\section{INTRODUCTION}

Anastomotic insufficiency in the postoperative period after esophageal surgery is the most feared complication and is associated with high morbidity and mortality rates. The incidence varies greatly, with studies reporting it to be up to $60 \%$, and it correlates with factors such as surgical experience, case load, and general health of the patient. ${ }^{1,2}$ The consequences of

\section{Received: March 3, 2021 Revised: June 7, 2021}

Accepted: June 21, 2021

Correspondence: Nader El-Sourani

Department for General and Visceral Surgery, University Hospital, Klinikum Oldenburg AöR, Rahel-Strauss Straße 10, Oldenburg 26133, Germany

Tel: +49-441-403-77628, Fax: +49-441-403-3413,E-mail: el-sourani.nader@ klinikum-oldenburg.de

ORCID: https://orcid.org/0000-0002-6146-7785

(cc) This is an Open Access article distributed under the terms of the Creative Commons Attribution Non-Commercial License (http://creativecommons.org/ licenses/by-nc/3.0) which permits unrestricted non-commercial use, distribution, and reproduction in any medium, provided the original work is properly cited. anastomotic insufficiency include high risk of postoperative mortality and prolonged intensive care unit and hospital stay, which subsequently lead to increased hospital costs and negative long-term outcomes, such as worse long-term survival and quality of life. ${ }^{3-5}$

Insufficiency of the esophagogastrostomy can be managed with surgical exploration or conservative management through nil per mouth, antibiotics, and drainage. ${ }^{6}$ However, as soon as an operative revision is performed, mortality rates increase and can exceed $60 \%{ }^{7}$ During the last decades, different non-operative measures have played an increased role in the treatment of anastomotic insufficiency following esophageal surgery. Of these measures, endoscopic treatment procedures are the most important to mention, including endoscopic clipping, injection of fibrin glue, and implantation of self-expanding plastic stents (SEPS) and self-expanding metal stents (SEMS). ${ }^{8-10}$ Recently, endoluminal vacuum therapy (EVT) has been introduced to treat defects of the upper gastrointestinal tract. EVT was first described by Weidenhagen for controlling 
anastomotic leakage during rectal surgery. ${ }^{11}$ The same procedure was adopted for defects in the upper gastrointestinal tract in $2006 .^{12}$

To date, however, the treatment of anastomotic insufficiency remains controversial, as the indications for conservative, endoscopic, or surgical treatment remain non-standardized. ${ }^{13,14}$ Recently, the Surgical Working Group on Endoscopy and Ultrasound (CAES) developed a classification for intrathoracic anastomotic leaks, suggesting a classification and treatment algorithm. ${ }^{15}$

The aim of this study was to evaluate the endoscopic treatment options for postoperative intrathoracic anastomotic leaks, mainly comparing SEMS and EVT in a tertiary university center and outlining a more standardized approach for the future.

\section{MATERIAL AND METHODS}

\section{Study Population}

Between 2010 and 2020, all patients who underwent Ivor Lewis esophagectomy for an underlying malignancy were included in this study. All patients who developed post-surgical anastomotic leaks were further analyzed. This led to a total of 28 patients who were treated for anastomotic leak at our hospital. In addition, the following parameters were examined: date of surgery, postoperative day of detection of an anastomotic leak, CAES classification, type of treatment used, median combined intensive care unit and intermediate care stay, median hospital stay, tumor histology, tumor grading, neoadjuvant therapy, number of lymph nodes harvested, R-status, operation method, operation time, morbidity, and mortality. Regarding endoscopic treatment, the following variables were analyzed: location of the defect, size of the defect, number of stents or vacuum sponges used, event-related complications, length of treatment, and treatment outcome.

\section{Diagnosis of Anastomotic Insufficiency}

An anastomotic leak was defined as a communication between the intra- and extraluminal compartments through a defect in the integrity of the intestinal wall of the anastomosis. Routine examination using a dynamic swallow study was performed until 2016. If the dynamic swallowing study suggested the presence of an anastomotic leak, it was followed by an upper endoscopy (UE) or computed tomography (CT). After 2016, routine examination of the anastomosis was abolished and was only performed if patients showed symptoms suggestive of a leak. If so, a combination of UE and CT was performed. Subsequently, patients with macroscopic visible mediastinal leakage cavity (referred to as the "extraluminal cavity") were always treated using EVT. In contrast to this group, patients with smaller anastomotic defects and none or small leakage cavity (called "intraluminal cavity") were treated with stent therapy. In addition, EVT has become increasingly established as a standard therapy over the past few years.

\section{EVT}

If an anastomotic leakage was clinically suspected or confirmed using a CT scan, EVT was evaluated as a therapeutic option. A sufficient external thoracic drainage was inserted in the case of a huge mediastinal septic abscess. In cases of confirmed or suspected anastomotic leakage, UE was performed in sedated or mostly intubated patients (GIF-H180, GIF-H190; Olympus Co., Tokyo, Japan). If there was evidence of a large extraluminal leakage cavity, which could only be inspected using a small-caliber nasal endoscope (GIF-N180) but not with a normal endoscope (GIF-H180, GIF-H190), the defect was expanded using balloon dilatation (CRE ${ }^{\mathrm{TM}}$ Wireguided $12-15$ mm; Boston Scientific, Marlborough, MA, USA). During the initial endoscopy, the leakage cavity was cleaned and measured to determine the required length and diameter of the sponge, which was then reshaped accordingly. Open-pore polyurethane sponges, Eso-SPONGE ${ }^{\circledR}$ (B. Braun Melsungen AG, Melsungen, Germany) with a primary diameter of $24 \times 55 \mathrm{~mm}$ and a $12 \mathrm{CH}$ Redon drain or an individually adapted sponge (V.A.C. VERAFLO ${ }^{\mathrm{TM}}$ Dressing Kit; KCI, St. Paul, USA) fixed to a drain (Argyle ${ }^{\mathrm{TM}}$ Edlich Gastric Lavage Tube; $16 \mathrm{CH}$, Medsitis, USA), were used.

In general, the intraluminal placement of the sponge in the case of small anastomotic defects (usually less than 8 to 10 $\mathrm{mm}$ ) or residual cavities with no infection can be differentiated from the intracavitary placement of the sponge, where it is introduced through the wall defect into the extraluminal, i.e., mediastinal, cavity. The intracavitary version of EVT was preferred. For placement of the sponge, two endoscopic methods were used, the "push" technique or the "piggyback" technique. Using the push technique, the sponge was advanced to the correct location along an overtube with a pusher or the endoscope, and a specially approved device (Eso-SPONGE ${ }^{\circledR}$; B. Braun Melsungen AG, Melsungen, Germany). Using the piggyback technique, the sponge was placed in the leakage cavity under direct endoscopic vision, while a suture loop placed at the tip of the sponge was grasped using endoscopic forceps and the sponge was pulled close to the endoscope. While the first technique is often used for small anastomotic leaks with intraluminal positioning of the sponge, the second technique is preferably used for intracavitary placement of the sponge. The drainage tube was placed transnasally and connected to a variable-speed medical vacuum pump (V.A.C. ULTA ${ }^{\circledR}$; KCI, San Antonio, Texas, USA). Suction was applied at a negative 
pressure of 75-125 $\mathrm{mmHg}$. In addition, a transnasal gastric or duodenal tube was inserted for enteral nutrition. After a dwell time of 3-5 days, the next endoscopy was performed. In this procedure, the sponge was removed orally after it was disconnected from the vacuum pump. The treated cavity was then examined using an endoscope to document the success of the treatment, particularly with a focus on subsequent granulation. A new sponge was inserted after re-measuring the size of the cavity to determine the size of the new sponge. To promote effective cavity closure, the diameter of the sponge was first reduced without reducing its length to allow for closure of the remaining channel in subsequent treatment cycles from the distal part of the channel to the proximal part. EVT was continued until the cavity was reduced to less than $1 \mathrm{~cm}$. During each endoscopy, the CAES grading of anastomotic insufficiency in the esophagus was reevaluated retrospectively.

Figure 1 illustrates the typical clinical course of treatment in one of the study patients undergoing successful EVT.

\section{SEMS}

Similar to the principles of EVT, endoscopic evaluation was carried out with regard to the size of the defect, existence of an extraluminal leak cavity, and perfusion of the anastomosis or the gastric sleeve. SEMS were mostly inserted under direct endoscopic view in the intensive care unit (ICU) without radiological control. First, a stiff wire (Amplatz Super Stiff Guidewire; Boston Scientific) was placed down to the stomach under endoscopic control. A fully or partially covered SEMS with a diameter of 22-28 mm was inserted and released under endoscopic view (Wall Flex ${ }^{\mathrm{TM}}$ Esophageal Stent, partially covered, 22-28 mm, Boston Scientific) with a total length of 100 $\mathrm{mm}$. The SEMS was removed after approximately three weeks. In the case of persistent insufficiency, another stent was inserted. CAES grading was reevaluated retrospectively.

Figure 2 illustrates the management of an anastomotic leak in one of the study patients using an SEMS.

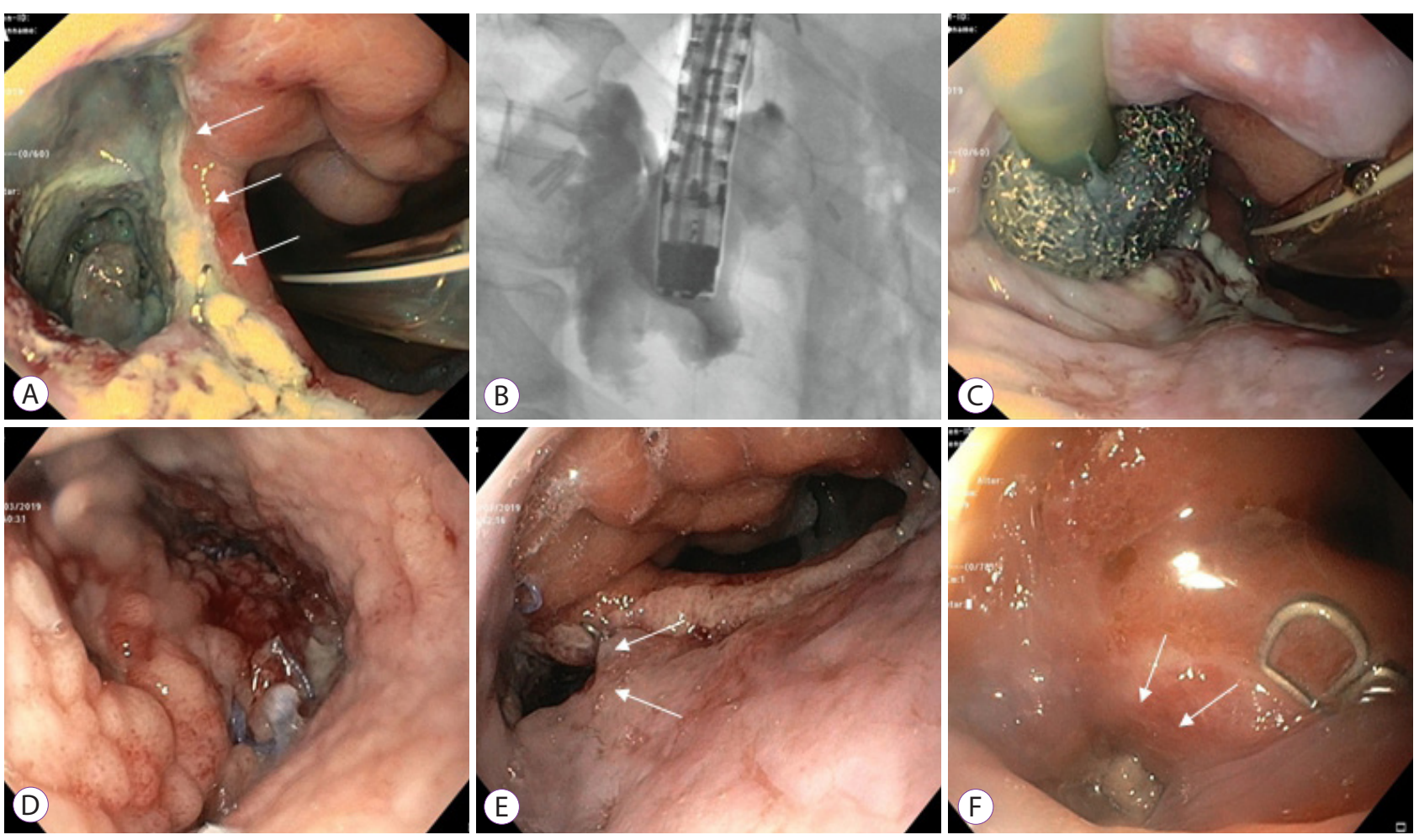

Fig. 1. Management of anastomotic leakage by endoluminal vacuum therapy (EVT). A 75-year-old patient with ypT3 pN1 M0 R0 Barrett's carcinoma treated with thoracoabdominal esophagectomy after neoadjuvant chemotherapy. (A, B) An approximately 3-cm large anastomotic leakage cavity on the 10th postoperative day in which deeper fistulas could be excluded. (C, D) After EVT for 10 days, there was a clear tendency towards granulation. (E) The insufficiency cavity was significantly smaller and the EVT was completed after 14 days. (F) Endoscopic control after a total of 24 days showed an almost closed insufficiency. 


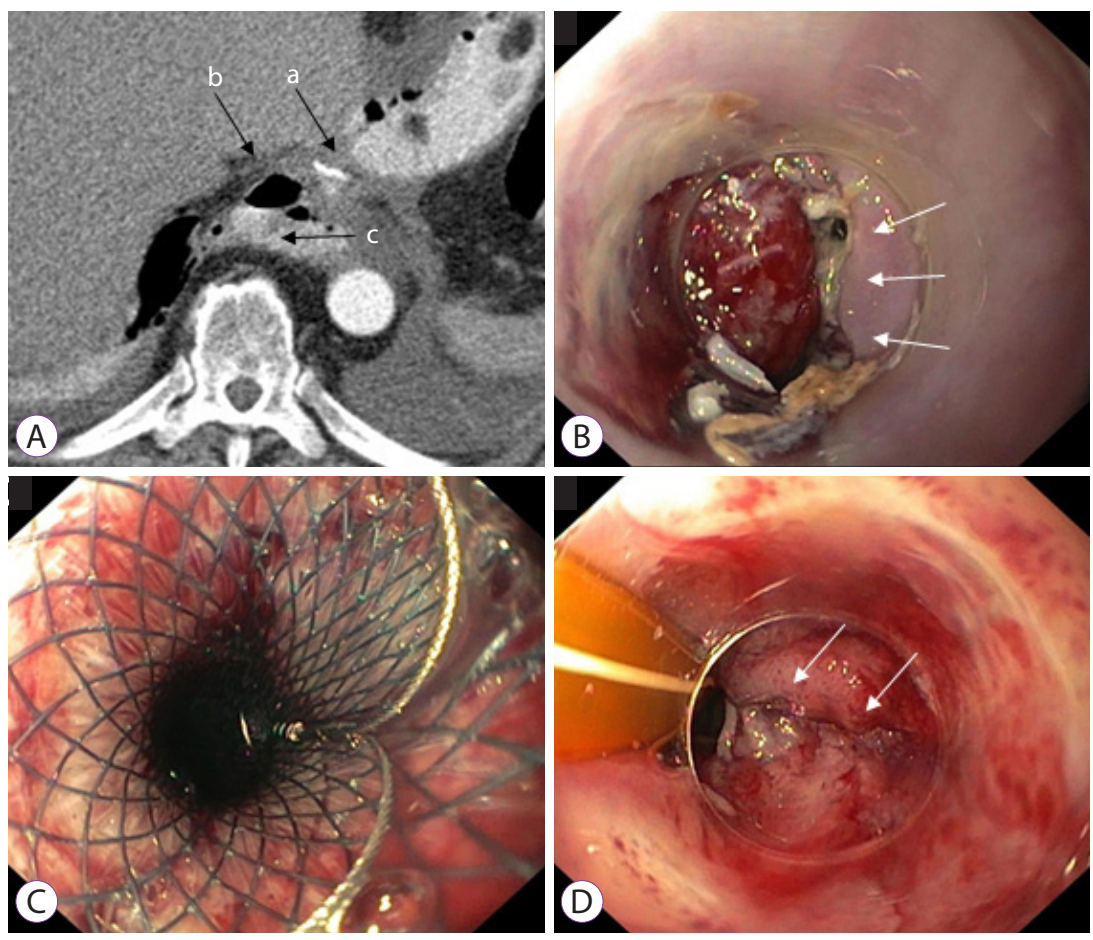

Fig. 2. Management of anastomotic leakage using a self-expanding metal stent (SEMS). A 67-year-old patient with $\mathrm{pT} 1 \mathrm{~b}$ (sm1) pN0 M0 R0 adenocarcinoma was treated with transhiatal distal esophagectomy. (A) CT scan in the region of the anastomosis (a) with detection of extraluminal free air (b) and contrast medium (c) on the 7th postoperative day. (B) Endoscopy with detection of an approximately 6-mm small anastomotic leakage with an otherwise vital anastomosis. (C) In the absence of evidence for an abscess, a fully covered self-expanding metal stent with a diameter of $28 \mathrm{~mm}$ was implanted. (D) The stent was removed after three weeks. The small anastomotic leakage was almost completely closed.

\section{Statistical Analysis}

Statistical analysis was performed using IBM SPSS Statistics Version 24 64-Bit-Version for Mac OS (IBM Co., Armonk, NY, USA). Continuous variables are presented as medians. To compare these variables, we employed analysis of variance (ANOVA) with multiple factors. Categorical variables were compared using the chi-squared test. Statistical significance was defined as $p<0.05$.

\section{RESULTS}

\section{Patient Characteristics}

Between 2010 and 2020, 141 patients underwent Ivor Lewis esophagectomy for underlying malignancy. All relevant patient characteristics are presented in Table 1 . A total of 28 patients were diagnosed with postoperative anastomotic leakage, resulting in an anastomotic insufficiency rate of $19.9 \%$. Of these patients, three patients were treated conservatively, 13 patients were treated with EVT, seven patients were treated with SEMS, one patient was treated with a hemoclip, and four patients received primary surgery to treat the defect (Fig. 3). Six patients required surgical revision after the initiation of endoscopic treatment. All 28 patients were classified using the CAES classification.
Of the 28 patients, 23 were men and five were women. The median age was 58.5 years (range: $32-75$ years). The median body mass index and American Society of Anesthesiologists classification were $25 \mathrm{~kg} / \mathrm{m}^{2}$ and 2, respectively. The reasons for esophagectomy were adenocarcinoma in 25 patients $(89.3 \%)$ and squamous cell carcinoma in three cases (10.7\%). Twenty of the 28 patients received neoadjuvant chemotherapy or radiotherapy. All but one patient underwent open Ivor Lewis esophagectomy with a median operation time of $290 \mathrm{~min}$ (range: 144-624 min).

\section{Overall Clinical Outcomes}

Of the 141 patients, 28 (19.9\%) were diagnosed with postoperative anastomotic insufficiency. The median time from surgery to diagnosis was 7.5 days (range: $2-30$ days). The median distance from the upper incisor to the defect was 25 $\mathrm{cm}$ (range: 18-30 cm), with a median defect size of $10 \mathrm{~mm}$ (range: 5-30 mm). In 10 patients, the defect developed into a macroscopic visible extraluminal cavity. The median hospital stay was 48.5 days (range: 9-193 days) with a median ICU/intermediate care (IMC) stay of 22 days (range: 9-193 days). Of the 28 patients who developed anastomotic insufficiency, 20 (71.4\%) were treated successfully, while eight (28.6\%) patients died. The overall endoscopic findings are presented in Table 2. Eight patients were treated with alternative methods to EVT 
Table 1. Patient Characteristics

\begin{tabular}{|c|c|}
\hline Sex & \\
\hline Male & $23(82.1)$ \\
\hline Female & $5(17.9)$ \\
\hline Age & $58.5(32-75)$ years \\
\hline Tumor type & \\
\hline Adenocarcinoma & $25(89.3)$ \\
\hline Squamous cell carcinoma & $3(10.7)$ \\
\hline Neoadjuvant therapy & \\
\hline No therapy & $8(18.6)$ \\
\hline Neoadjuvant therapy & $20(71.4)$ \\
\hline Open surgery & $27(96.4)$ \\
\hline Laparoscopic assisted surgery & $1(3.6)$ \\
\hline Operation time & $290(144-625) \mathrm{min}$ \\
\hline Lymph nodes harvested & $19.5(6-37)$ \\
\hline R0 & $28(100)$ \\
\hline Grade & \\
\hline Grade 1-2 & $17(60.7)$ \\
\hline Grade 3 & $11(39.3)$ \\
\hline Combined ICU/IMC stay & 22 (9-193) days \\
\hline Hospital stay & 48.5 (9-193) days \\
\hline
\end{tabular}

Data are presented as the number (\%) or median (range).

ICU, intensive care unit; IMC, intermediate care.

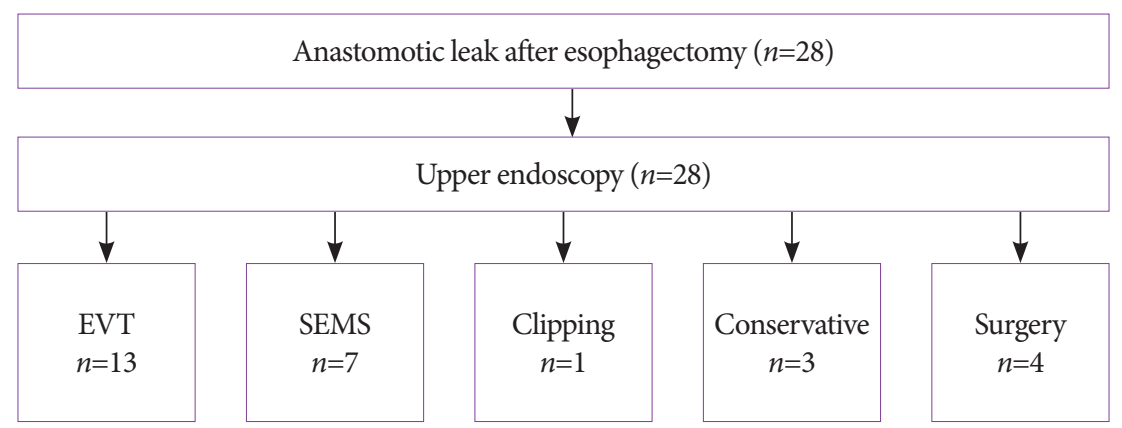

Fig. 3. Treatment algorithm for patients treated for anastomotic leak after esophagectomy. Of the 28 patients, 13 were treated with endoluminal vacuum therapy (EVT), seven with self-expanding metal stents (SEMS), one with a clip, three were treated conservatively, and four patients required surgery to treat the defect.

or SEMS. Of these eight patients, four underwent primary surgery, two of whom required additional surgical revision and died postoperatively. Three of the eight patients were treated conservatively, and one patient was treated with endoscopic clipping of the defect. All patients receiving non-surgical al- ternative treatments were successfully treated. The median combined IMC/ICU and hospital stay were 12 and 29 days, respectively. The median defect size was $3.5 \mathrm{~mm}$ (range: $2-4$ $\mathrm{mm}$ ) for the conservative group and $22.5 \mathrm{~mm}$ (range: 10-25 $\mathrm{mm}$ ) for the surgical group. 
Table 2. Overall Endoscopic Findings

\begin{tabular}{lc}
\hline Defect size & $10(4-30) \mathrm{mm}$ \\
Defect location & $25(18-30) \mathrm{cm}$ \\
Intraluminal & $18(64.3)$ \\
Extraluminal cavity & $10(35.7)$ \\
CAES grading & $3(10.7)$ \\
I & $15(53.6)$ \\
II & $8(28.6)$ \\
IIIa & $2(7.1)$ \\
IIIb & $13(46.4)$ \\
Endoluminal vacuum therapy & $7(25)$ \\
Self-expanding metal stent therapy & $1(3.6)$ \\
Clipping of the defect &
\end{tabular}

Data are presented as the number (\%) or median (range).

CAES, The Surgical Working Group on Endoscopy and Ultrasound.

\section{Clinical Outcomes: EVT}

Thirteen patients (46.4\%) with a mediastinal leakage cavity (extraluminal cavity) were treated with EVT. The median time to diagnosis of anastomotic leak after the primary surgery was 8 days. All patients with an extraluminal cavity were treated using EVT, and all of those within the EVT group who died had an extraluminal cavity. No EVT-related complications were observed. The median time for EVT was 24.5 days (range: 8-80 days) with a median of five exchanged sponges (range: 4-18). The median defect size was $15 \mathrm{~mm}$, and the median defect was located $26 \mathrm{~cm}$ from the upper incisor. Eight patients were successfully treated (61.5\%), while five patients passed away. However, complete closure of the defect was achieved in 12 patients (92.3\%). Five patients needed surgical revision during EVT, and 4 of those patients died. All of these patients required surgery due to complications that could not be attributed to EVT. The reasons for surgery were ischemia of the colon, necrosis of the pancreas, postoperative incarcerated hiatal hernia, and hemothorax. The median ICU/IMC stay was 38 days (range: 9-193 days), with a median hospital stay of 74 days (range: 9-193 days). The overall outcomes of EVT are shown in Table 3.

\section{Clinical Outcomes: SEMS}

Seven patients (25\%) were treated with SEMS. In the stent group, none of the patients had an extraluminal cavity. The median duration of SEMS therapy was 22 days (range: 3-31 days), with a median of one SEMS exchange (range: 1-2). The median defect size was $6 \mathrm{~mm}$, and the median defect was lo- cated $23 \mathrm{~cm}$ from the upper incisor. Six patients were successfully treated (85.7\%), while one patient required surgical revision and died. The median ICU/IMC stay was 20 days (range, 16-57 days) with a median hospital stay of 41 days (range: 22-123 days). The median time to diagnosis of anastomotic leak was 7 days. During SEMS treatment, event-related complications, including stent migration $(n=1)$ and perforation $(n=1)$, were noted. A close defect was achieved in six out of seven patients (85.7\%). The overall outcomes of SEMS therapy are shown in Table 3.

\section{Comparison of SEMS and EVT}

Statistical analysis to compare SEMS and EVT revealed that there was a statistical significance $(p<0.05)$ in terms of defect size, presence of an intraluminal or extraluminal cavity, and sponges/stents used. No other difference in outcomes between the two treatment options were statistically significant. The results of the statistical analysis comparing the two modalities are presented in Table 3.

\section{DISCUSSION}

Post-surgical anastomotic insufficiency is one of the most feared complications and is associated with high morbidity and mortality rates. Therefore, the goal of every physician involved in the treatment of patients following esophageal surgery is to diagnose and manage the event and its related complications in a timely manner. As already mentioned, the 
Table 3. Outcomes of Endoluminal Vacuum Therapy and Self-Expanding Metal Stent Therapy

\begin{tabular}{lccc}
\hline & $\begin{array}{c}\text { EVT group } \\
(\boldsymbol{n}=\mathbf{1 3})\end{array}$ & $\begin{array}{c}\text { SEMS group } \\
(\boldsymbol{n}=7)\end{array}$ & Statistical analysis \\
\hline Time to diagnose insufficiency & $8(5-30)$ days & $7(6-11)$ days & $\mathrm{F}=1.992 ; p>0.05$ \\
Defect size & $15(4-30) \mathrm{mm}$ & $6(5-20) \mathrm{mm}$ & $\mathrm{F}=4.561 ; p<0.05$ \\
Defect location & $26(20-30) \mathrm{cm}$ & $23(20-28) \mathrm{cm}$ & $\mathrm{F}=3.004 ; p>0.05$ \\
CAES classification & & 0 & $\chi^{2}=4.929 ; p>0.05$ \\
I & 0 & 6 & \\
II & 8 & 0 & \\
IIIa & 4 & 1 & \\
IIIb & 1 & 0 & $\chi^{2}=10.769 ; p<0.05$ \\
Intraluminal & 3 & $\chi^{2}=10.769 ; p<0.05$ \\
Extraluminal cavity & 10 & $22(3-31)$ days & $\mathrm{F}=1.392 ; p>0.05$ \\
Duration of EVT & $24.5(8-80)$ days & $1(1-2)$ & $\mathrm{F}=12.047 ; p<0.05$ \\
Number of procedures/patient & $5(4-18)$ & $6(85.7)$ & $\chi^{2}=0.220 ; p>0.05$ \\
Complete closure & $12(92.3)$ & $2(28.6)$ & $\chi^{2}=0.196 ; p>0.05$ \\
Procedure-related complications & $0(0)$ & $20(16-57)$ days & $\mathrm{F}=3.210 ; p>0.05$ \\
Combined ICU/IMC stay & $38(9-193)$ days & $41(22-123)$ days & $\mathrm{F}=3.890 ; p>0.05$ \\
Hospital stay & $74(9-193)$ days & & \\
\hline
\end{tabular}

Data are presented as number (\%) or median (range).

CAES, The Surgical Working Group on Endoscopy and Ultrasound; EVT, endoluminal vacuum therapy; ICU, intensive care unit; IMC, intermediate car; SEMS, self-expanding metal stents.

incidence of anastomotic leaks after surgery can be up to $50 \%$ and is associated with a mortality rate of $20 \%$. However, if operative revision is necessary, the mortality rate can exceed $60 \%$. Historically, the damage was controlled through a combination of surgery and conservative management with nil per mouth, antibiotics, and drainage. In the last decade, however, EVT and SEMS have been used to successfully manage intrathoracic anastomotic insufficiency. In 2018, the German CAES group suggested a classification and treatment algorithm for intrathoracic leaks. Here, surgical revision was only suggested in cases of graft necrosis or in patients with pre-sepsis. ${ }^{15}$

Endoscopic therapy for intrathoracic leaks includes clipping of the defect, use of EVT, and insertion of different types of stents. Schaheen et al. performed a systematic review of the use of stents in the management of anastomotic leaks and found 25 studies. ${ }^{16}$ Endoscopic placement was successful in $72 \%$ of patients, with an overall mortality of $15 \%$. The types of stents used included SEMS and SEPS, with an average time remaining in situ of 6 and 8 weeks, respectively. Stent-related complications included stent migration, perforation, bleeding, and tissue ingrowth. These findings mirror the results of our retrospective analysis. SEMS treatment was successful in $85.7 \%$ of the patients, with a mortality rate within the group of $14.3 \%$. Two out of seven patients had event-related complications, including stent migration (14.3\%) and perforation (14.3\%). However, due to the small sample size, the results were not statistically significant $(p>0.05)$. The median number of stents used was one. The systematic review concluded that endoscopic stenting remains an experimental therapy as stenting has the ability to "stent the seal" but not "heal the leak"; therefore, mortality remains high even after endoscopic stenting.

EVT is another option for treating anastomotic leaks. Similar to wound vacuum for secondary wound infections, a sponge is placed intraluminally and intracavitarily with added suction through a transnasal drain. A systematic review of three available studies showed that 37 out of 40 patients (93\%) were successfully treated with EVT without the presence of EVT-related complications. ${ }^{17-19}$ This is also reflected in our results. In 12 out of 13 patients (92.3\%), complete closure of the leak was achieved with no EVT-related complications noted.

Unfortunately, five patients required surgery during EVT due to complications that could not be attributed to the therapy. The reasons for surgery were ischemia of the colon, necrosis of the pancreas, incarcerated hiatal hernia, and hemothorax. This is also reflected by the CAES classification (EVT vs. 
SEMS), as shown in Table 3. However, due to the small sample size, there was no statistical significance regarding CAES classification ( $p>0.05)$.

The difference in defect size between the SEMS and EVT groups was very noticeable in our retrospective analysis. There was a tendency to treat patients with smaller defects and no mediastinal leakage cavity with stent therapy. The defect size in the SEMS group was $6 \mathrm{~mm}$ compared to $15 \mathrm{~mm}$ in the EVT group, yielding a 2.5-fold size difference. A comparison of the defect size between the two groups using multivariate analysis was statistically significant $(p<0.05)$. In addition, all patients who had an extraluminal cavity on endoscopic findings were treated with EVT instead of SEMS $(p<0.05)$, and only patients in the EVT group had a worse outcome, underlying the importance of extraluminal, i.e., mediastinal, cavities for the overall prognosis. As already outlined, there was a tendency to treat more critically ill patients with EVT. These results suggest that SEMS treatment is only warranted in patients with a small defect size and with no extraluminal cavity. Unfortunately, we were not able to find similar results across published data of other groups analyzing EVT and SEMS therapy, as defect size and presence of an extraluminary cavity were not specifically described and analyzed. Only one study group mentioned the size of the defect and the presence of a cavity in their study. ${ }^{20}$ In our opinion, these are two crucial findings that seem to be related to treatment and patient outcomes and should be analyzed further.

This study has some limitations. First, this was a retrospective, non-randomized study. Second, the sample size was small and, therefore, it was difficult to compare the two treatment modalities to identify a statistically significant difference. However, although these limitations are present, it is one of the only available studies mentioning defect size and analyzing its potential outcome on the treatment option that should be chosen.

In summary, our results suggest that EVT seems to be a better treatment option for patients with a large defect size and the presence of an extraluminal cavity compared to SEMS. It can be safely applied to critically ill patients with large defects. SEMS therapy seems only warranted in non-septic patients with a small defect size and no extraluminal cavity. Primary surgical revision should be reserved only for septic patients with graft necrosis.

Conflicts of Interest

The authors have no potential conflicts of interest.

Funding

None.

\section{Author Contributions}

Conceptualization: Nader El-Sourani, Christian Meinhardt

Data curation: NES, Sorin Miftode, Maximilian Bockhorn, Alexander Arlt, CM

Formal analysis: NES, SM, MB, AA, CM

Investigation: NES, SM, MB, AA, CM

Methodology: NES, CM

Project administration: NES, CM

Resources: NES

Software: NES

Supervision: $\mathrm{MB}, \mathrm{AA}, \mathrm{CM}$

Validation: NES, MB, AA, CM

Visualization: NES, CM

Writing-original draft: NES

Writing-review\&editing: NES, MB, AA, CM

\section{ORCID}

Nader El-Sourani

Sorin Miftode

Maximilian Bockhorn

Alexander Arlt

Christian Meinhardt https://orcid.org/0000-0002-6146-7785 https://orcid.org/0000-0003-3071-8702 https://orcid.org/0000-0002-7027-6606 https://orcid.org/0000-0002-6160-1059 https://orcid.org/0000-0002-6801-4076

\section{REFERENCES}

1. Ryan CE, Paniccia A, Meguid RA, McCarter MD. Transthoracic anastomotic leak after esophagectomy: current trends. Ann Surg Oncol 2017;24:281-290.

2. El-Sourani N, Bruns H, Troja A, Raab H-R, Antolovic D. Routine use of contrast swallow after total gastrectomy and esophagectomy: is it justified? Pol J Radiol 2017;82:170-173.

3. Hölscher AH, Vallböhmer D, Brabender J. The prevention and management of perioperative complications. Best Pract Res Clin Gastroenterol 2006;20:907-923.

4. Karl RC, Schreiber R, Boulware D, Baker S, Coppola D. Factors affecting morbidity, mortality, and survival in patients undergoing Ivor Lewis esophagogastrectomy. Ann Surg 2000;231:635-643.

5. Singh D, Maley RH, Santucci T, et al. Experience and technique of stapled mechanical cervical esophagogastric anastomosis. Ann Thorac Surg 2001;71:419-424.

6. Michel L, Grillo HC, Malt RA. Operative and nonoperative management of esophageal perforations. Ann Surg 1981;194:57-63.

7. Kuehn F, Loske G, Schiffmann L, Gock M, Klar E. Endoscopic vacuum therapy for various defects of the upper gastrointestinal tract. Surg Endosc 2017;31:3449-3458.

8. Hünerbein M, Stroszczynski C, Moesta KT, Schlag PM. Treatment of thoracic anastomotic leaks after esophagectomy with self-expanding plastic stents. Ann Surg 2004;240:801-807.

9. Truong S, Böhm G, Klinge U, Stumpf M, Schumpelick V. Results after endoscopic treatment of postoperative upper gastrointestinal fistulas and leaks using combined Vicryl plug and fibrin glue. Surg Endosc 2004;18:1105-1108.

10. Doniec JM, Schniewind B, Kahlke V, Kremer B, Grimm H. Therapy of anastomotic leaks by means of covered self-expanding metallic stents after esophagogastrectomy. Endoscopy 2003;35:652-658.

11. Wiedenhagen R, Spelsberg F, Lang RA, et al. New method for sepsis control caused by anastomotic leakage in rectal surgery: the Endo-VAC. Colorectal Dis. 2003;5:1-4.

12. Loske G, Schorsch T, Müller CT. Endoskopische Vakuumtherapie der akuten Mediastinitis: Ein modernes minimal-invasives Therapieverfahren. Chirurg 2016;87:790-792. 
13. Raju GS, Tarcin O. Endoscopic management of anastomotic esophageal leaks. Techniques in Gastrointestinal Endoscopy 2006;8:66-71.

14. Pross M, Manger T, Reinheckel T, Mirow L, Kunz D, Lippert H. Endoscopic treatment of clinically symptomatic leaks of thoracic esophageal anastomoses. Gastrointest Endosc 2000;51:73-76.

15. Schaible A, Schmidt T, Diener M, et al. Intrathoracic anastomotic leakage following esophageal and cardial resection: definition and validation of a new severity grading classification. Chirurg 2018;89:945-951.

16. Schaheen L, Blackmon SH, Nason KS. Optimal approach to the management of intrathoracic esophageal leak following esophagectomy: a systematic review. Am J Surg 2014;208:536-543.

17. Schniewind B, Schafmayer C, Voehrs G, et al. Endoscopic endoluminal vacuum therapy is superior to other regimens in managing anastomotic leakage after esophagectomy: a comparative retrospective study. Surg Endosc 2013;27:3883-3890.

18. Schorsch T, Müller C, Loske G. Endoscopic vacuum therapy of anastomotic leakage and iatrogenic perforation in the esophagus. Surg Endosc 2013;27:2040-2045

19. Weidenhagen R, Hartl WH, Gruetzner KU, Eichhorn ME, Spelsberg F, Jauch KW. Anastomotic leakage after esophageal resection: new treatment options by endoluminal vacuum therapy. Ann Thorac Surg 2010;90:1674-1681.

20. Jeon JH, Jang HJ, Han JE, et al. Endoscopic vacuum therapy in the management of postoperative leakage after esophagectomy. World J Surg 2020;44:179-185. 